\title{
Non-local mean value image de-noising algorithm based on self-adaption
}

\author{
Bin Yang ${ }^{1}$, Mingming $\mathrm{Guo}^{2}$, Xinhua Dou ${ }^{3}$ \\ ${ }^{1}$ Hefei University Department of computer science and technology, Hefe 230601, China \\ ${ }^{2}$ Department of Hefei University of Technology Academic, Hefei 23009, China \\ ${ }^{3}$ School of Computer and Information, Hefei University of Technology, Hefe 23009, China
}

Keywords: Image de-noising; Non-local means; Information of directional structure; filter parameters; search neighbor-hood.

\begin{abstract}
In traditional non-local mean value algorithm, both filter parameters and measurement of search neighborhood are constant globally and it does not show diverse structural features of different areas in images, which makes the weight of similarity of image blocks distributed unreasonably, and thus de-noising can be not effective. In order to avoid that, a non-local mean value image de-noising algorithm based on self-adaption is pro-posed. With analysis of the content of image blocks, image blocks from different areas will obtain different filter parameters and search fields, which cause the similarity weights of image blocks a more proper distribution. Experimental results showed that the new algorithm, after de-noising, achieved an increase of peak signal to noise ratio of images and, at the same time, reserved details of images and marginal information effectively.
\end{abstract}

\section{Introduction}

In 2006, Buades and others using self-similar image exists in a large number of these redundant information presented non-local mean (NLM) algorithm $[1,4,8,10]$. After that, many scholars through the study of the filtering parameters, made a number of improved algorithm. Coupe et al. by modeling for $h^{2}=f\left(\sigma^{2},\left|N_{i}\right|, \beta\right)$ think that the filtering parameters not only related to the noise variance and the number of pixels in the neighborhood of the relevant [3]. And they proposed a method for the quantitative estimation of filter parameters in the literature [11]. Salmon et al. also studied the influence of the central image block weight distribution and the size of the search area for non-local means algorithm [9]. The vast majority of these methods are based on a global approach to identify a fixed filter parameters, using a fixed filter parameters to filter. The image in real life is complex and changeable[6,13], using a unified image content filtering parameters and practical differences that do not match, do not have adaptability, the denoising effect must have an effect. Brox et al. is often difficult to find a pair of images for a global optimal filtering parameters and size of the area for hidden, so that each part of the image can achieve better de-noising effect [2]. After a number of experiments, the filter parameters and for hidden area size and content of the image noise and have a great relationship, so the use of a self- adaptive filtering parameters and selection methods for hidden area of more reasonable size, it will get better de-noising effect $[5,7,12]$. Experiments show that the algorithm has a good de-noising effect while still able to retain the details of the image and the edge of the structural information, then obtain better de-noising effect.

\section{Non-local means denoising algorithm}

The purpose of denoising is obtained noise-free image from noisy images. Non-local means to compute a full picture of all the pixels as a weighted average of the estimated value of the point after de-noising pixels:

$$
N L[v](i)=\sum_{j \in \Omega} w(i, j) v(j)
$$


Here, the right value ${ }^{w(i, j)}$ depends on the similarity between $i$ and ${ }^{j}$, and the condition is satisfied, $0<w(i, j)<1, \sum_{j} w(i, j)=1$

Similarity between pixels $i$ and $j$ is determined by the pixel Gaussian weighted Euclidean distance, as follows:

$$
d(i, j)=\left\|v\left(N_{i}\right)-v\left(N_{j}\right)\right\|_{2, \alpha}^{2}
$$

The weights are defined by the formula (3)

$$
w(i, j)=\frac{1}{Z(i)} e^{-\frac{d(i, j)}{h^{2}}}
$$

Filtering parameters $h$ control the depth of the filter, if the value is large, the image is de-noised too smooth, many details will be lost, so the image becomes more blurred; if the value is small, the filter is not sufficient to effectively remove noise, image de-noised still left a lot of noise, de-noising poor. Filter parameters should select a more appropriate value, both to ensure the effective removal of noise, not too smooth image, able to retain the details of the image.

\section{Non-local means algorithm adaptive filtering parameters}

Filter parameter selection. Traditional non-local means filtering algorithm using global parameters in a fixed manner, regardless of the image structures that are more complex content, or relatively simple structure relative to a single image, are used with a filter parameter value. The differences in approach and image content does not match the facts, cannot meet the filter parameters using a larger flat area in the image, use a smaller filter parameters requires a relatively complex structure of the region. Filtering parameters should be determined according to the specific content of the image block .In other words, the filter parameters should be adaptive.

Image structure feature extraction. Minutiae parts of the image is generally reflected in the edges of the image, the image gray level pixel value difference is more obvious. Before the image de-noising, you first need to image edge detection, distinguishing different features of the image shown in Figure 1:
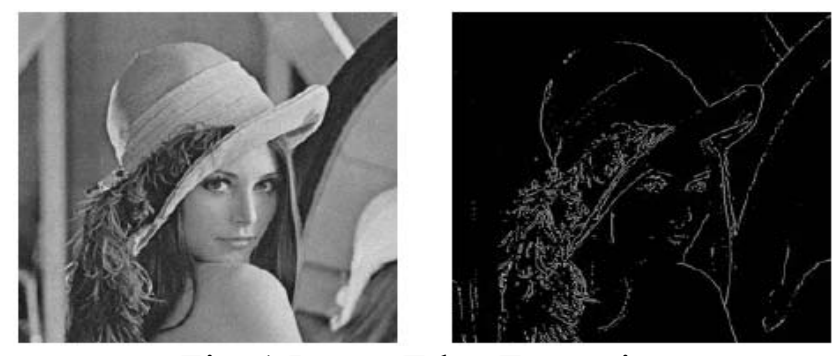

Fig. 1 Image Edge Extraction

By Image Edge Extraction method to distinguish between the different areas of the image where the pixels to determine the center of the image pixel image block is in the details at the edges of the image. If the center of the image block of pixels at the edges, like the details of the image, you should use a smaller filter parameter values, if not in, then use a larger filter parameter values. In this way the choice of filter parameters in different regions of the image block to obtain different filter parameters, so that each image block to obtain its best filter parameters, you can achieve better filtering effect.

Direction of the image block structure information. The direction of the image block structure information to reflect changes in gray value of the image block, and it also reflects the structural characteristics of the image, through the direction of the structure of the image blocks extracted up images from the flat area to the complex structure of the region over the process. Direction of the image information of the block structure shown in Figure 2:

Select an image block search area. Non-local means algorithm in the search area is a region in image block similarity measure between the need to search for in the search area for all image blocks gives some weight, similar to the image block will receive greater weight, similarity smaller for a 
larger weights. All right image block value and the entire search area is 1 . All tiles will get a right value, even those that are not similar to the image block will have a certain weight. Large search area may get more similar tiles, this situation is conducive to de-noising, but will also bring more dissimilar image block. These rights are not similar to the value obtained image block add up, it will have to de-noising impact cannot be ignored. While a smaller search area, cannot be more similar to the image block, this will weaken the denoising. Thus, the size of the search area should be determined according to where the pixels in the image de-noising with specific location, rather than using a fixed global uniform value.

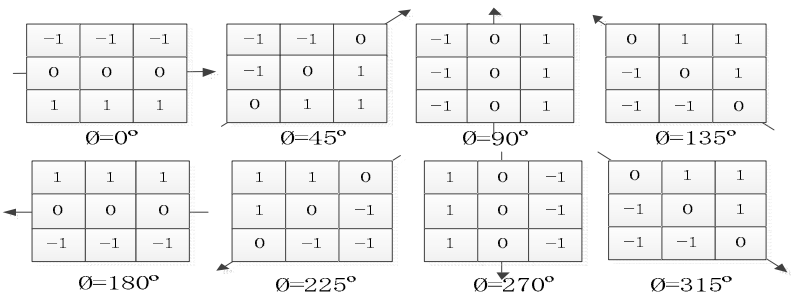

Fig. 2 Block diagram of the image orientation information

By extracting the edge features of the image and the direction information extracting structure, to determine the pixel location of the image point position of the belt and the de-noising filter parameters to determine the search area, determined by a large number of experiments, the filter parameters and the search area may be as follows:

(1) An edge feature if the pixels are not to be de-noised image, image blocks where no apparent direction structure information, the filter parameters can be set $30 \sigma$, the search area size $27 \times 27$

(2) an edge feature if the pixels are not to be de-noised image, but where the image block having a clear orientation structure information, the filter parameters can be set $25 \sigma$, the search area size $21 \times 21$.

(3) If the edge to be de-noised pixels in the feature image, image blocks where no apparent direction structure information, the filter parameters can be set $20 \sigma$, the search area size $15 \times 15$.

(4) If the edge to be de-noised pixels in the feature image, where the image block has a clear direction of structural information, the filter parameters can be set $15 \sigma$, the search area size $9 \times 9$.

\section{Analysis of experimental results}

Through experiments renderings and peak signal to noise ratio for both image denoising effect after analysis and comparison, which can be found in the non-local means algorithm uses adaptive filtering parameters and the search area (ANLM) is better than the traditional NLM.

Experimental results chart analysis.
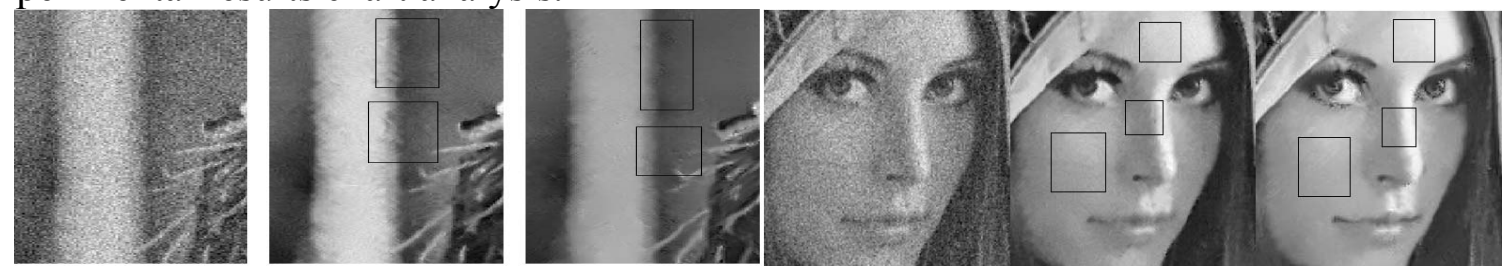

Fig. 3 De-noising comparison chart

From the figure 3 relatively flat area of the face, improved de-noising algorithm is obviously superior to the non-local means algorithm, which is due to the image of the face is a relatively flat area of the filter parameters and the use of larger search area, so that noise removal is more complete, able to find more similarities with the image block, so that the pixel to be de-noised obtain a more accurate gray value. Which contrast can be found in the complex area of the image, the more efficient use of the filter parameters and the search area, the details of structural features can effectively retain the image so that the image de-noised more complete.

Comparison of PSNR. MSE and PSNR commonly used in image quality and objective evaluation methods. Peak signal to noise ratio is calculated using the experimental images obtained by two different methods, the results shown in Table 1, we can see that the peak signal to noise ratio 
improved non-local means algorithm is higher than traditional non-local means algorithm. PSNR each graph and the corresponding take ten times the average.

Table 1 SNR resulting in varying degrees of noise

\begin{tabular}{ccccc}
\hline Image Name & $\alpha=10$ & $\alpha=15$ & $\alpha=20$ & $\alpha=30$ \\
\hline Camer (NLM) & 34.76 & 33.31 & 32.78 & 31.01 \\
Camer (ANLM) & 35.89 & 34.88 & 33.98 & 32.13 \\
House(NLM) & 35.04 & 34.29 & 33.56 & 31.97 \\
House(ANLM) & 35.87 & 35.01 & 34.87 & 32.10 \\
Rice(NLM) & 34.63 & 33.44 & 32.81 & 31.69 \\
Rice(ANLM) & 35.85 & 34.78 & 34.03 & 32.19 \\
\hline
\end{tabular}

\section{Summary}

Through in-depth study of the non-local means filtering algorithm parameters and the search area, and analyzes the impact of adaptive filtering parameters and the search area for de-noising effect, combined with the characteristics of the different characteristics of the image itself contains a structure, an adaptive filter parameters and non-local means algorithm for searching the neighborhood, and gives the method for determining the filter parameters and search neighborhood. Experiments show that the algorithm by de-noising and PSNR are superior to traditional NLM.

\section{Acknowledgment}

Supported by Talents Science Research Foundation of Hefei University (13RC02).

\section{References}

[1] Buades A,ect al.A review of image denosing algo rithms, with a new one [J]. Multiscale Modeling and Simulation, 2006, 4(2):490-530.

[2] Brox T,Cremers D.Iterated nonlocal means for texture restoration. Proceedings of the 1st Internation Conference on Scale Space and Variatioal Methods in Computer Vision, 2007, 4485:13-24.

[3] Coupe P,Yger P,Prima .An optimized blockwise nonlocal menas denoining filter for 3-D magnetic resonance images IEEE Transaction on Medical imaging,2008,27(4):425-411

[4] Deledalle C,Salmon J.Image denosing with patch based PCA:local means global.In BMVC, Britain(2011).

[5] Di Hong W, Liu X.. Acta Photonic Sinic, 2006, 35(5):766-771.

[6] Gonzalez, Digital Image Processing (Second Edition) [M], 2003.

[7] Jiang G,Wang X,ect al. Overview on image quality assessment[J]. Journnal of Electronics \& Information Technology, 2010, 32(1):219-226

[8] Mahmoudi M,Sapio G.Fast image and video denosing via non-local means of similar neighborhoods. IEEE Single process, 2005, lett 12:839 -842.

[9] Salmon J.On two parameters for denoising with nonlocal means. IEEE Singal Processing Letters, 2010, 17(3):269-272.

[10]Weng F non-local bilateral filter for image denosing. ICA (2010), PP. 34-257.

[11]Wang Zhiming, Zhang adaptive fast non-local denoising algorithm. Chinese Journal of Image and Graphics, 2009, 14(4):669-675.

[12] Wang Z ,Bovik A,ect al.Image equality assessment from errorvisibiliy to Structural similarity [J]. IEEE Trans on Image Processing, 2004, 13(4): 600-612.

[13]Xue Xi year, MATLAB applications [M] in the digital signal processing Beijing: Tsinghua University Press.2003. 